\title{
From the Desk of the Editor
}

\section{K. Harish ${ }^{1}$}

Published online: 20 March 2017

(C) Indian Association of Surgical Oncology 2017

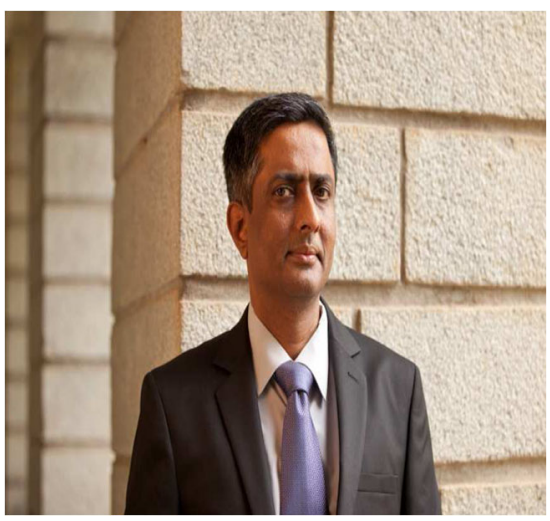

Uro-Oncology has progressed as a separate niche area in Oncology. Advances in terms of surgical techniques, technological advances with laparoscopes and robotics added to the diagnostics make it a separate field of expertise. Improvements in understanding of cancer as a disease and its behaviour have a huge bearing on evaluation and management of these cancers. This issue has a conservative surgerythe nephron sparing surgery for renal cell carcinoma for early disease and the management of venacaval thrombosis for the advanced disease. Another interesting area is radical prostatectomy for the locally advanced disease. In addition, this issue has some interesting articles which could also raise a debate like the reverse axillary mapping.

Over the last few years, the quality of the manuscripts has improved. However, the aim of the Journal is to provide 'Indian' data. We would encourage authors to collate data from centres in their areas of expertise and publish. We hope that we would receive more such manuscripts which could mirror or contradict western data.
K. Harish

drkhari@yahoo.com

1 M.S. Ramaiah Medical College, Bengaluru, India 